\title{
Erratum
}

Food Sci. Biotechnol. 19: 575-582 (2010). DOI 10.1007/s10068-010-0081-3

\section{Chemistry of Bread Aroma: A Review}

\section{Cho IH and Peterson DG.}

Correspondence to: dgp@umn.edu

Erratum in: Food Sci. Biotechnol. 22: 1479 (2013). DOI: 10.1007/s10068-013-0240-4

Since the publication of this article, the authors have discovered small errors in odorants and odor description of Table 3.

The correct Table 3 is shown here.

Table 3. Potent odorants of wheat bread crumb $(\mathrm{FD} \geq 32)^{1)}$

\begin{tabular}{cllc}
\hline No. & \multicolumn{1}{c}{ Odorants } & Odor description ${ }^{2)}$ & FD $^{3)}$ \\
\hline 1 & (E)-2-Nonenal & Green, tallowy & 512 \\
2 & (E,E)-2,4-Decadienal & Fatty, waxy & 512 \\
3 & Trans-4,5-epoxy-(E)-2-decenal & Metallic & 512 \\
4 & (Z)-2-Nonenal & Green & 128 \\
5 & 1-Octen-3-one & Mushroom-like & 128 \\
6 & Diacetyl & Buttery & 64 \\
7 & Methional & Cooked potato-like & 64 \\
8 & 2,4-Decadienal & Fatty, green & 64 \\
9 & 3-Methylbutanol & Malty & 32 \\
10 & 2-Acetyl-1-pyrroline & Roasty & 32 \\
11 & (E,E)-2,4-Nonadienal & Fatty, waxy & 32 \\
12 & Unknown & Sulphurous & 32 \\
\hline
\end{tabular}

${ }^{1)}$ From Schieberle and Grosch (22)

${ }^{2)}$ Description of the odors recognized during GC-sniffing from the crumb of wheat bread

${ }^{3)}$ Flavor dilution 